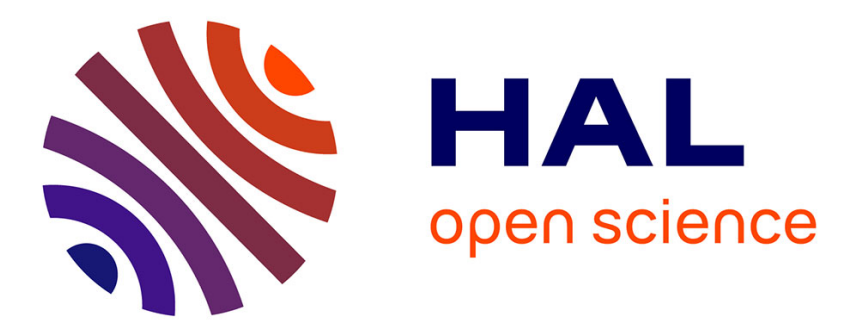

\title{
Phenomenological analysis of the singularities of the free energy near the freezing temperature of a spin glass in an applied field
}

\author{
J. Seiden
}

\section{- To cite this version:}

J. Seiden. Phenomenological analysis of the singularities of the free energy near the freezing temperature of a spin glass in an applied field. Journal de Physique Lettres, 1984, 45 (16), pp.807-812. 10.1051/jphyslet:019840045016080700 . jpa-00232415

\section{HAL Id: jpa-00232415 https://hal.science/jpa-00232415}

Submitted on 1 Jan 1984

HAL is a multi-disciplinary open access archive for the deposit and dissemination of scientific research documents, whether they are published or not. The documents may come from teaching and research institutions in France or abroad, or from public or private research centers.
L'archive ouverte pluridisciplinaire HAL, est destinée au dépôt et à la diffusion de documents scientifiques de niveau recherche, publiés ou non, émanant des établissements d'enseignement et de recherche français ou étrangers, des laboratoires publics ou privés. 
Classification

Physics Abstracts

64.60C

\title{
Phenomenological analysis of the singularities of the free energy near the freezing temperature of a spin glass in an applied field
}

\section{J. Seiden}

Institut d'Electronique Fondamentale (*), Université Paris XI, Bât. 220, 91405 Orsay Cedex, France

(Reçu le 20 février 1984, révisé le 2 mai, accepté le 19 juin 1984)

Résumé. - Nous tentons de déduire des conséquences observables des singularités mesurées de la susceptibilité non linéaire d'un verre de spin au voisinage de sa température de gel. On calcule les singularités de la chaleur spécifique et de la section efficace de diffusion des neutrons. On développe la susceptibilité $\chi(t, H)=\frac{\mathrm{d} M}{\mathrm{~d} H}=\sum(2 n+1) a_{n} t^{-\Gamma_{n}} H^{2 n}$ et l'on déduit des inégalités entre les $\Gamma_{n}$. On discute les conditions de validité et les implications possibles de lois d'échelle. Les relations déduites concernant la susceptibilité sont en accord avec l'expérience.

\begin{abstract}
We deduce some observable consequences associated with the measured singularities of the non-linear susceptibility in spin glasses near the freezing temperature. Singularities of the specific heat and of the neutron diffusion cross-sections are pointed out. The susceptibility is expanded as $\chi(t, H)=\frac{\mathrm{d} M}{\mathrm{~d} H}=\sum(2 n+1) a_{n} t^{-\Gamma_{n}} H^{2 n}$ and inequalities between the $\Gamma_{n}$ are derived under plausible assumptions. If approximate scaling holds, experimental implications of it are discussed. All the deduced expressions for the susceptibility are in agreement with experiment.
\end{abstract}

\section{Introduction.}

The mean field theory of spin glasses [1, 2] predicts for reduced temperatures $t$ such that $0<t \ll 1$ (where $t=\frac{T-T_{\mathrm{f}}}{T_{\mathrm{f}}}, T_{\mathrm{f}}$ being the freezing temperature in zero field) an equilibrium magnetization $N M(t, H)=N g \mu_{\mathrm{B}} S(t, H)$ given by

$$
M(t, H)=\chi(t, 0) H+g \mu_{\mathrm{B}}\left[a_{1} t^{-1} H^{3}+a_{2} t^{-3} H^{5}+\cdots\right] .
$$

$N$ is the total number of spins, $H$ the applied field. Recent measurements [3] carried out on several spin glasses [AgMn 10.6\%, $\underline{\mathrm{CuMn} 1 \%}, \mathrm{Gd}_{37} \mathrm{Al}_{63}, \mathrm{Al}_{2} \mathrm{Mn}_{3} \mathrm{Si}_{3} \mathrm{O}_{12},\left(\mathrm{MnF}_{2}\right)_{0.65}\left(\mathrm{BaF}_{2}\right)_{0.15}$

(*) Laboratoire associé au CNRS. 
$\left.\left(\mathrm{NaPO}_{3}\right)_{0.2}, \ldots\right]$ have shown that

$$
S(t, H)-a_{0} H=\sum_{n=1}^{\infty} a_{n} t^{-\Gamma_{n}} H^{2 n+1},
$$

with $a_{0}=\chi(t, 0) / g \mu_{\mathrm{B}}$. The $\Gamma_{n}$ are critical exponents. For instance, in amorphous manganese aluminosilicate, $\Gamma_{1} \simeq 3.1 ; \Gamma_{2} \simeq 7.6$ [4].

Previously, Suzuki [2] has worked out a phenomenological theory of spin glasses near $T_{\mathrm{f}}$. He expands the free energy in powers of $q$, the Edwards-Anderson order parameter, and then asserts that the singular part of this energy does contain a scaling function $f\left[q\left(T-T_{\mathrm{f}}\right)^{-\beta}\right]$ as in classical phase transitions. From this assumption, scaling laws result for $M(t, H)$ and $C(t, H)$. But $q$ is not the right order parameter and perhaps, it may not be relevant [5].

Here, therefore, discarding the framework of phase transition theory, we chose another route : taking for granted, from experiment, the existence of the first singular term $a_{1} t^{-\Gamma_{1}} H^{3}$ in the expansion (2), we try to deduce some consequences concerning the static properties of the system in the neighbourhood $t>0$ of the point $t=0, H=0$. We shall not refer to any order parameter. Our analysis does not suppose the existence of some phase transition at $T_{\mathrm{f}}$. If a phase transition occurs, our results extend to $t=0$. If there is no static transition, our results will be fairly approximate except in the vicinity of the point $t=0, H=0$; the meaning of $T_{\mathrm{f}}$, a well defined temperature in all measurements quoted above, has then to be clarified.

We shall derive inequalities between the $\Gamma_{n}$ which are verified in all experiments, and investigate the conditions required for scaling to hold. Among them, low H values will be shown to be necessary. Applications including specific heat and neutron diffusion are briefly discussed.

We suppose that all points $t \geqslant 0, H \neq 0$ belong to the region where the system is ergodic and where no phase transition occurs. We suppose that all measured thermodynamic quantities have their true equilibrium values. Our analysis applies only to the region $t \geqslant 0, H \neq 0$.

\section{Inequalities between the $\Gamma_{n}$.}

We consider the non-linear susceptibility per spin

$$
\begin{gathered}
\chi_{\mathrm{s}}(t, H)=\chi(t, H)-\chi(t, 0), \\
N \chi_{\mathrm{s}}(t, H)=\beta g^{2} \mu_{\mathrm{B}}^{2} \sum_{i, j}\left[\left\langle\overline{S_{i}^{z} S_{j}^{z}}\right\rangle_{H}-\left\langle\overline{S_{i}^{z} S_{j}^{z}}\right\rangle_{0}-\left\langle\overline{\left.S_{i}^{z}\right\rangle_{H}\left\langle S_{j}^{z}\right.}\right\rangle_{H}\right] .
\end{gathered}
$$

The suffix $H$ recalls that the mean values are calculated in the applied field $H$ (directed along $\mathrm{O} z$ ), the suffix $\mathbf{0}$ refers to zero field. $\mathbf{S}_{i}$ is the spin on the site $\mathbf{R}_{i}$. The upperlines are averages over the locations of the sites $\mathbf{R}_{i}$.

We will show first, under plausible assumptions, that if $\Gamma_{1}>0$, the critical exponents $\Gamma_{n}$ obey

$$
\Gamma_{n} \geqslant n \Gamma_{1} \text {. }
$$

We use

$$
S(t, H)-a_{0} H=\frac{1}{g \mu_{\mathrm{B}}} \int_{0}^{H} \mathrm{~d} H^{\prime} \chi_{\mathrm{s}}\left(t, H^{\prime}\right) .
$$

We introduce a correlation radius $r_{\mathrm{c}}(t, H)$ such as-if $\left|\mathbf{R}_{i}-\mathbf{R}_{j}\right|>r_{\mathrm{c}}(t, H)$ then, for all directions $\mathbf{R}_{i}-\mathbf{R}_{j}$, one has

$$
\left\langle\overline{S_{i}^{z} S_{j}^{z}}\right\rangle_{H}-\left\langle\overline{S_{i}^{z} S_{j}^{z}}\right\rangle_{0} \simeq\left\langle\overline{\left.S_{i}^{z}\right\rangle_{H}\left\langle S_{j}^{z}\right.}\right\rangle_{H}
$$


and a function $\Lambda(t, H)$ defined by

$$
\sum_{j}\left[\left\langle\overline{S_{i}^{z} S_{j}^{z}}\right\rangle_{H}-\left\langle\overline{S_{i}^{z} S_{j}^{z}}\right\rangle_{0}-\left\langle\overline{\left.S_{i}^{z}\right\rangle_{H}\left\langle S_{j}^{z}\right.}\right\rangle_{H}\right] \equiv \Lambda(t, H) S^{2}(t, H) .
$$

$\Lambda(t, H)$ is some effective number of spins correlated to any one, say $\mathbf{S}_{i}$. We suppose that $\Lambda(t, H)$ may be expanded in powers of $H$ as

$$
\Lambda(t, H)-b_{0} t^{-\Lambda_{0}} \equiv \Lambda_{\mathrm{s}}(t, H)=\sum_{n=1}^{\infty} b_{n} t^{-\Lambda_{n}} H^{2 n}
$$

It is implied that in the expansions (2) and (9), only the most divergent terms, $t^{-\Gamma_{n}}$ and $t^{-A_{n}}$ respectively, are retained for each power of $H$. If we use (9) and (2) to calculate (8) and then (6), by equating the coefficients of $H^{2 n+3}$ on the two sides of equation(6), one finds

$$
\sum_{\substack{p, q \\ p+q \leqslant n}} b_{n-p-q} a_{p} a_{q} t^{-\Lambda_{n-p-q}-\Gamma_{p}-\Gamma_{q}} \propto a_{n+1} t^{-\Gamma_{n+1}} .
$$

Taking $n=0$ in (10), it follows that $b_{0} \neq 0, \Lambda_{0}=\Gamma_{1}$. Then

$$
\Gamma_{n+1}=\max \left\{\Lambda_{n-p-q}+\Gamma_{p}+\Gamma_{q}\right\}
$$

where the maximum must be found by varying the integers $p$ and $q$ with $p+q \leqslant n, p \geqslant 0$, $q \geqslant 0$.

By (11)

$$
\Lambda_{0}+\Gamma_{n-p}+\Gamma_{p} \leqslant \Gamma_{n+1}
$$

so that $\Gamma_{2} \geqslant 2 \Gamma_{1}$. By induction, one finds $\Gamma_{n} \geqslant n \Gamma_{1}$. In the same manner, it may be shown that for $n \geqslant 2$

$$
\begin{aligned}
& \Gamma_{2 n} \geqslant n\left(\Gamma_{1}+\Gamma_{2}\right), \\
& \Gamma_{2 n-1} \geqslant n \Gamma_{1}+(n-1) \Gamma_{2} .
\end{aligned}
$$

An infinite sequence of inequalities may thus be derived by successively considering $\Gamma_{3}, \Gamma_{4}, \ldots$

\section{Specific heat.}

The equilibrium magnetization is given by $-g \mu_{\mathrm{B}} S(t, H)=\partial F(t, H) / \partial H, F(t, H)$ being the free energy per spin,

$$
F(t, H)-F(t, 0)+\frac{\chi(t, 0)}{2} H^{2}=-g \mu_{\mathrm{B}} \sum_{n=1}^{\infty} \frac{a_{n}}{2 n+2} t^{-\Gamma_{n}} H^{2 n+2} .
$$

The specific heat per spin $C(t, H)=-T \partial^{2} F / \partial T^{2}$ is thus

$$
C_{\mathrm{s}}(t, H) \equiv C(t, H)-C(t, 0)-\frac{1}{2}\left(\frac{\partial^{2} C}{\partial H^{2}}\right)_{0} H^{2}=\frac{T}{T_{\mathrm{f}}^{2}} g \mu_{\mathrm{B}} \sum_{n=1}^{\infty} \frac{\Gamma_{n}\left(\Gamma_{n}+1\right)}{2 n+2} a_{n} t^{-\Gamma_{n}-2} H^{2 n+2} .
$$

The RHS is singular, the lowest order singular term being proportional to $t^{-\Gamma_{1}-2} H^{4}[6]$. The $t^{-\Gamma_{1}-2} H^{4}$ singularity has by now been observed by performing measurements of the magnetocaloric effect in CuMn $0.25 \%$ [7]. 


\section{Neutron diffusion.}

In the quasi-static approximation, the differential cross-section for the diffusion of neutrons by $N$ identical spins $\mathbf{S}_{i}$ [with no orbital contribution to the localized magnetic moment $g \mu_{\mathrm{B}} \mathbf{S}_{i}$ ] for $k \rho \ll 1$ is

$$
\frac{\mathrm{d} \sigma}{\mathrm{d} \Omega} \propto \sum_{v}\left(1-K_{v}^{2}\right) \sum_{i, j} \overline{\exp i \mathbf{k}\left(\mathbf{R}_{i}-\mathbf{R}_{j}\right)\left\langle S_{i}^{v} S_{j}^{v}\right\rangle_{H}},
$$

$\mathbf{k}$ is the transferred moment, $k=|\mathbf{k}|, K=\mathbf{k} / k . v$ refers to Cartesian components. $\rho$ is the spatial extension of the magnetic ions. We have supposed that $\mathbf{k}$ is directed along one of the axis $\mathrm{Ox}, \mathrm{O} y$ or $\mathrm{O} z$. The direct interaction between the neutron magnetic moment and the applied field as also the magnetic form factors of the ions have been neglected. Equation (16) supposes that the mean energy transfers in the neutron-system collisions are low compared to the energy of the incident neutrons [8]. If three measurements are made with $\mathbf{k}$ equal successively to $\mathbf{k}_{x}, \mathbf{k}_{y}, \mathbf{k}_{z}$ directed respectively along $\mathrm{O} x, \mathrm{O} y, \mathrm{O} z$, if the corresponding cross-sections are called $\left(\frac{\mathrm{d} \sigma}{\mathrm{d} \Omega}\right)_{x},\left(\frac{\mathrm{d} \sigma}{\mathrm{d} \Omega}\right)_{y},\left(\frac{\mathrm{d} \sigma}{\mathrm{d} \Omega}\right)_{z}$, then, if $k r_{\mathrm{c}}(t, H) \ll 1$, and if $\left|\mathbf{k}_{x}\right| \simeq\left|\mathbf{k}_{y}\right| \simeq\left|\mathbf{k}_{z}\right|=k$

$$
\begin{aligned}
\left(\frac{\mathrm{d} \sigma}{\mathrm{d} \Omega}\right)_{x}+\left(\frac{\mathrm{d} \sigma}{\mathrm{d} \Omega}\right)_{y}-\left(\frac{\mathrm{d} \sigma}{\mathrm{d} \Omega}\right)_{z} \propto & \left\{\sum_{i, j}\left[\left\langle\overline{S_{i}^{z} S_{j}^{z}}\right\rangle_{H}-\left\langle\overline{\left.S_{i}^{z}\right\rangle_{H}\left\langle S_{j}^{z}\right.}\right\rangle_{H}\right]+\right. \\
& \left.+\sum_{i, j} \frac{\overline{\exp i \mathbf{k}_{x}\left(\mathbf{R}_{i}-\mathbf{R}_{j}\right)+\exp i \mathbf{k}_{j}\left(\mathbf{R}_{i}-\mathbf{R}_{j}\right)}}{2}\left\langle S_{i}^{z}\right\rangle_{H}\left\langle S_{j}^{z}\right\rangle_{H}\right\} .
\end{aligned}
$$

The first RHS terms between brachets, equal to $N \chi(t, H) / \beta g^{2} \mu_{\mathrm{B}}^{2}$, is the incoherent contribution. The second RHS term is the Bragg contribution, here it is blurred around $k=0$ by the disorder of spin positions and the fluctuations of local magnetization $\left\langle S_{i}^{z}\right\rangle$. Its order of magnitude is $N c k^{-3}\left(\delta R^{2} / R^{2}\right) S^{2}(t, H)$ where $\overline{\delta R^{2}} / R^{2}$ denotes symbolically the relative disorder of spin positions and where the fluctuations of $\left\langle S_{i}^{z}\right\rangle$ have been neglected. $c$ is the number of spins per unit volume. Both terms are singular.

\section{Scaling.}

Measurements performed on several spin glasses have shown that approximately

$$
\frac{\chi_{\mathrm{s}}(t, H)}{g \mu_{\mathrm{B}}}=\sum_{n=1}^{\infty}(2 n+1) a_{n} t^{-\Gamma_{n}} H^{2 n} \approx t^{\Gamma-\Gamma_{1}} f\left(\frac{H^{2}}{t^{\Gamma}}\right)
$$

for low values of $t$ and $H$. We shall investigate the conditions required for scaling to hold.

5.1. - Because there is no transition on the line $t=0, H \neq 0$,

$$
\lim _{t \rightarrow 0} \chi_{\mathrm{s}}(t, H)=\chi_{\mathrm{s}}(0, H), \lim _{t \rightarrow 0} C_{\mathrm{s}}(t, H)=C_{\mathrm{s}}(0, H)
$$

remain finite for $H \neq 0$. By construction, $\chi_{s}(0,0)=0, C_{s}(0,0)=0$. Consider $\chi_{s}$ and suppose that $\chi_{s}(0, H)$ may be expanded as

$$
\left(g \mu_{\mathrm{B}}\right)^{-1} \chi_{\mathrm{s}}(0, H)=\sum_{i=1}^{\infty} d_{i}\left(H^{2}\right)^{\Delta_{i}}, \quad \Delta_{i}>\Delta_{i-1}>\cdots>\Delta_{1}>0 .
$$


Thus, there must be a field $H_{0}$ such that if $H<H_{0}$

$$
\left(g \mu_{\mathrm{B}}\right)^{-1} \chi_{\mathrm{s}}(0, H) \approx d_{1} H^{2 \Delta_{1}}, \quad H<H_{0} .
$$

$\Delta_{i}$ depends upon the $a_{j}$ and $\Gamma_{j}$. We put

$$
g \mu_{\mathrm{B}}\left(\frac{\chi_{\mathrm{s}}(t, H)}{t^{\Gamma-\Gamma_{1}}}\right)^{-1}=\phi\left(\frac{H^{2}}{t^{\left(\Gamma-\Gamma_{1}\right) \mid \Delta_{1}}}, t\right) .
$$

We shall see that $\Gamma-\Gamma_{1}=\Gamma_{2}-2 \Gamma_{1} \geqslant 0$; thus, $\phi$ remains finite and continuous if $t \rightarrow 0$. If $t \approx 0^{+}$and $0<H<H_{0}$,

$$
g \mu_{\mathrm{B}}\left(\frac{\chi_{\mathrm{s}}(t, H)}{t^{\Gamma-\Gamma_{1}}}\right)^{-1} \approx \phi\left(\frac{H^{2}}{t^{\left(\Gamma-\Gamma_{1}\right) / \Delta_{1}}}, 0\right) \approx d_{1}^{-1}\left(\frac{H^{2}}{t^{\left(\Gamma-\Gamma_{1}\right) / \Delta_{1}}}\right)^{-\Delta_{1}}
$$

which is scaling, but only for $t \approx 0^{+}$.

5.2. - We now show that scaling may be extended to values $0<t \ll 1$ and that

$$
\Gamma=\Gamma_{2}-\Gamma_{1}, \Delta_{1}=\frac{\Gamma_{2}-2 \Gamma_{1}}{\Gamma_{2}-\Gamma_{1}}
$$

We shall first prove the following lemma : $\chi_{\mathrm{s}}(t, H)$ scales as in (18) with $\Gamma \neq \Gamma_{1}$ if and only if $\chi_{\mathrm{s}}(t, H)$ and $\Lambda_{\mathrm{s}}(t, H)$ display the same singular structure in their respective developments (18) and (9), more precisely if

$$
\Lambda_{n}=\Gamma_{n}+\Gamma, \Gamma>\Gamma_{1} .
$$

Equation (25) means that the theory contains only one singular function determined by the infinite sequence of exponents $\Gamma_{1}, \Gamma_{2}, \ldots$

The theorem is proved by induction.For $n=1 \mathrm{in}(10)$, we have at the LHS the terms $b_{0} a_{0} a_{1} t^{-2 \Gamma_{1}}$ and $b_{1} a_{0}^{2} t^{-\Gamma^{1}-\Gamma}$ and by (11) and (25), $\Gamma_{2}=\Gamma_{1}+\Gamma$, and so on. Finally, $\Gamma_{n}=\Gamma_{1}+(n-1) \Gamma$. Because of (5), one cannot have $\Gamma<\Gamma_{1}$. Conversely, if there exist an integer $p \geqslant 2$ such that $\Lambda_{p}=\Gamma_{p}+\Delta, \Delta \neq \Gamma$ but if $\Lambda_{n}=\Gamma_{n}+\Gamma$ for $n<p$, one finds $\Gamma_{p+1}=2 \Gamma_{1}+(p-1) \Gamma$ if $\Delta<\Gamma_{1}$, and $\Gamma_{p+1}=\Gamma_{1}+(p-1) \Gamma+\Delta$ if $\Delta>\Gamma_{1}$. Thus, no scaling law may hold.

This proof shows also that the scaling term on the RHS of (18) represents only the most divergent contribution to $\chi_{\mathrm{s}}(t, H)$ and that one has

$$
\chi_{\mathrm{s}}(t, H) \propto t^{\Gamma-\Gamma_{1}} f\left(\frac{H^{2}}{t^{\Gamma}}\right)\left[1+\mathcal{O}\left(t^{\Gamma-\Gamma_{1}}\right)\right] .
$$

Because scaling holds for $t \approx 0^{+},(25)$ must be satisfied, which in turn implies that scaling holds for $0<t \ll 1$. The identification $\Gamma=\left(\Gamma-\Gamma_{1}\right) / \Delta_{1}$ results in the second equation (24). In mean field theory, $\Gamma_{1}=1, \Gamma_{2}=3$ thus, $\Delta_{1}=1 / 2$ and $\chi_{s} \propto|H|$ in agreement with the Toulouse and Gabay [1] result.

From (26), it follows that

$$
\Delta_{n} \leqslant \frac{n\left(\Gamma_{2}-2 \Gamma_{1}\right)}{\Gamma_{2}-\Gamma_{1}}
$$

showing that scaling may only hold for low values of $H$. 
In the same manner, one finds

$$
C_{\mathrm{s}}(t, H)=t^{2 \Gamma-\Gamma_{1}-2} g\left(\frac{H^{2}}{t^{\Gamma}}\right), \quad C_{\mathrm{s}}(0, H) \propto H^{\frac{2}{\Gamma}\left(2 \Gamma-\Gamma_{1}-2\right)}
$$

so that, by continuity

$$
2 \Gamma_{2}-3 \Gamma_{1}-2 \geqslant 0 .
$$

The inequalities (5), (25), (29) are verified in all measurements.

\section{Discussion.}

If there is no static phase transition at $T_{\mathrm{f}}$, work on a more fundamental level beyond mean field theory would be required to explain why scaling has been observed near $T_{\mathrm{f}}$ and to clear up the physical meaning of this temperature which has been unambiguously defined in all experiments quoted above. In this case, our analysis remains approximately valid except in the vicinity of $t=0, H=0$, because our starting point, stated in section 1 , is an experimental result. Equation (21) and the second equation (28) strictly become false; (21) is then only an intermediate mathematical step, but (5), (25) and (29) remain true. (18) remains a fair approximation except near $t=0:$ as $t \rightarrow 0$, the rigorous equilibrium value of $\chi_{\mathrm{s}}(t, H)$ tends to depart from the RHS behaviour of (18). Identical conclusions may be reached for $C_{\mathrm{s}}(t, H)$ and $\mathrm{d} \sigma / \mathrm{d} \Omega$ : at $t=0, H=0$, all singularities have then vanished.

\section{Acknowledgments.}

We wish to thank P. Beauvillain, C. Chappert and J. P. Renard for numerous discussions and communication of their results before publication.

\section{References}

[1] Toulouse, G. and Gabay, M., J. Physique Lett. 42 (1981) L-103.

[2] Suzuki, M., Prog. Theor. Phys. 58 (1977) 1151.

[3] For a survey of recent experimental work on susceptibility singularities, see the report of J. P. Renard. "Transitions de phase dans les verres de spin. Les expériences sont-elles concluantes?" at the French Physical Society, Grenoble, Sept. 1983. "A travers la Physique " (Les Editions de Physique) 1984, p. 315.

[4] Beauvillain, P., Chappert, C., Renard, J. P., J. Physique Lett. 45 (1984) L-665.

[5] PARISI, G., J. Phys. A 13 (1980) 1101, 1574.

Thouless, D., De Almeida, J., Kosterlitz, J., J. Phys. C 13 (1980) 3272.

[6] See also Barbara, B., Malozemoff, A. P., Imry, Y., Phys. Rev. Lett. 47 (1981) 1852.

[7] Berton, A., Chaussy, J., Gandit, Ph., Odin, J., to be published. Their results were foreshadowed by Boyer, J. D., Fisher, R., Fogle, W. E., Phillips, N., Phys. Rev. Lett. 50 (1983) 1815.

[8] This condition is verified if $k_{\mathrm{B}} T_{\mathrm{N}} \gg|J| S, T_{\mathrm{N}}$ being the temperature of the thermal neutrons and $|J|$ the mean exchange integral between neighbouring spins in a Heisenberg spin glass. 